\title{
Smad7 maintains epithelial phenotype of ovarian cancer stem-like cells and supports tumor colonization by mesenchymal-epithelial transition
}

\author{
YIYING LI ${ }^{1 *}$, WENJIA GONG $^{1 *}$, XIAOLING MA ${ }^{1}$, XIAOXI SUN ${ }^{2}$, HUA JIANG $^{1,3}$ and TONG CHEN ${ }^{4}$ \\ ${ }^{1}$ Department of Gynecology, Obstetrics and Gynecology Hospital, Fudan University; ${ }^{2}$ Shanghai Ji Ai Genetics and \\ IVF Institute; ${ }^{3}$ Shanghai Key Laboratory of Female Reproductive Endocrine Related Diseases, Shanghai 200011; \\ ${ }^{4}$ Department of Hematology, Huashan Hospital, Fudan University, Shanghai 200040, P.R. China
}

Received January 31, 2014; Accepted August 7, 2014

DOI: $10.3892 / \mathrm{mmr} .2014 .2714$

\begin{abstract}
Epithelial ovarian carcinoma (EOC) is a lethal gynecological malignancy. Epithelial-mesenchymal transition (EMT) has an important role in the tumorigenesis and progression of EOC. During the process of EMT, the transforming grow th factor- $\beta$ (TGF- $\beta$ )-Smad signaling pathway has been indicated to regulate cell motility and tumor development. Among numerous EMT-associated transcripts, Smad7 is considered to be an inhibitor, however its involvement together with TGF- $\beta 1$ in the progression of ovarian cancer remains to be elucidated. The present study demonstrated that Smad7 was overexpressed in SK-OV-3 and stem-like side populations of EOC cells, both of which grow in an epithelial pattern. The transformation of cells from an epithelial to a mesenchymal phenotype was stimulated by TGF- $\beta 1$ with a corresponding increase in Smad7 expression in SK-OV-3 cells. These results indicate that $\mathrm{Smad} 7$ is a regulator in the maintenance of the epithelial phenotype in EOC cells, and may serve as an inhibitory element which targets TGF- $\beta$-stimulated EMT. Furthermore, inhibition of Smad7 resulted in cellular mesenchymal transformation, with an increased expression of $\mathrm{N}$-cadherin and a decreased expression of E-cadherin. The invasiveness and migratory capabilities of Smad7 small hairpin RNA transduced EOC cells was also reduced. The
\end{abstract}

Correspondence to: Dr Tong Chen, Department of Hematology, Huashan Hospital, Fudan University, 12 Wulumuqi Middle Road, Shanghai 200040, P.R. China

E-mail: chentong@fudan.edu.cn

Dr Hua Jiang, Department of Gynecology, Obstetrics and Gynecology Hospital, Fudan University, 419 Fangxie Road, Shanghai 200011, P.R. China

E-mail: jianghua@fudan.edu.cn

*Contributed equally

Key words: Smad7, TGF- $\beta 1$, epithelial-mesenchymal transition, metastasis, ovarian cancer findings of the present study have identified Smad7 as a fundamental factor in the maintenance of epithelial growth of EOC cells. Reversal of EMT results in a mesenchymal-epithelial transition, which is necessary for EOC cell colonization at metastatic sites.

\section{Introduction}

Epithelial ovarian cancer (EOC) is a lethal gynecological malignancy, the cells of which have highly aggressive and widely metastatic capabilities. The majority of patients with EOC are diagnosed at an advanced stage with extensive peritoneal metastases, leading to a high frequency of postoperative recurrence and mortality within five years (1). However, little is currently known about the underlying mechanisms of EOC tumor oncogenesis, invasiveness and metastasis.

The epithelial-mesenchymal transition (EMT) is a process constantly activated during tumor cell migration, invasion, intravasation and angiogenesis $(2,3)$. It is the biological process by which an epithelial cell transforms into a more motile mesenchymal cell, through the induction of mesenchymal markers including $\mathrm{N}$-cadherin and Vimentin (4). Whilst undergoing EMT, the cells lose their epithelial hallmarks, which are represented by E-cadherin-regulated cell-cell junctions and apical polarity (5). The cells start to exhibit a scattered and spindle-shaped mesenchymal morphology, reorganizing their cytoskeletons to facilitate migration and resist anoikis (5). The process of EMT is thought to be important to ovarian carcinogenesis and progression. It is associated with ovarian cancer peritoneal metastasis, as a consequence of the loosened contact of tumor cells with neighboring cells and disruption of the tissue architecture. These cellular changes enable the EOC cells to dissociate from the tumor mass and migrate to the peritoneal cavity (6-9). Furthermore, side population (SP) cells, which are considered to be cancer stem-like cells (CSCs), were identified as having an epithelial origin. Differentiation of SP cells into non-SP cells was previously demonstrated to be concomitant to the process of EMT and resulted in tumor cell heterogeneity, engraftment and progression (10). The reverse process of EMT, mesenchymal to epithelial transition (MET), has also been observed during both neoplasm development 
and tumor colonization $(7,11,12)$. It has been proposed that metastasizing tumor cells reverse the process of EMT, in order to implant at distant sites where the epithelial growth pattern is promoted (12-14).

During EMT, the TGF- $\beta$-Smad signaling pathway is associated with transcriptomic reprogramming (15). Regulating transcripts of EMT include: Smad1, Smad2, Smad3, Smad5 and Smad8, which are receptor-activated molecules; Smad4, a complex of Smad2 and Smad3 and a common-mediator; and Smad6 and Smad7, which are inhibitory proteins. It has previously been indicated that Smad7 represses the TGF- $\beta 1$ signaling pathway by combining with the activated TGF- $\beta 1$ receptor $(\mathrm{T} \beta \mathrm{RI})$ and preventing the phosphorylation of Smad2 and Smad3 (16). However, Smad7 has other effects, beyond its well-known inhibition of the canonical T $\beta$ RI-Smad pathway. It has heterogeneous functions in the progression of numerous cancers. In previous studies of nasopharyngeal carcinoma and cervical cancer, TGF- $\beta 1$ was shown to induce the overexpression of Smad7, which resulted in the malignant transformation and tumorigenicity $(17,18)$. In addition, the ectopic expression of Smad7 has been shown to block the progression of cancer in endometriosis (19). However, the association between Smad7 and TGF- $\beta 1$ stimulation in ovarian cancer EMT remains largely unknown.

In the present study, the expression of Smad7 during the process of EMT in different ovarian cancer cell lines was investigated. Analyzing the association between Smad7 and EOC pathogenesis may advance the understanding of the mechanisms behind the progression of EOC, and provide potential targets for anti-cancer therapy.

\section{Materials and methods}

Cell lines and culture conditions. ES-2, SK-OV-3 and OVCAR-3, human EOC cell lines, were obtained from American Type Culture Collection (Manassas, VA, USA). The HO-8910 and highly metastasizing HO-8910PM daughter line (20) EOC cells were purchased from the Cell Bank of the Shanghai Institute of Biochemistry and Cell Biology, (Shanghai, China). The cells were cultured in RPMI-1640 media (HyClone Laboratories Inc., Logan, UT, USA), supplemented with $10 \%$ fetal bovine serum (FBS; HyClone) and $1 \%$ penicillin/streptomycin (Gibco Life Technologies, Carlsbad, CA, USA). OVCAR-3 cells were maintained in RPMI-1640 containing 20\% FBS and $0.01 \mathrm{mg} / \mathrm{ml}$ bovine insulin (Sigma-Aldrich, St. Louis, MO, USA), according to the culturing guidelines. For TGF- $\beta 1$ stimulation, $10 \mathrm{ng} / \mathrm{ml}$ TGF- $\beta 1$ (R\&D Systems, Inc., Minneapolis, MN, USA) was added and the media was refreshed every $12 \mathrm{~h}$.

SP cell detection and selection. The procedure of Hoechst 33342 staining was performed as described by previous methods (21). Briefly, the cells were suspended at $1 \times 10^{6}$ cells $/ \mathrm{ml}$ in Dulbecco's modified Eagle's medium, containing $2 \% \mathrm{FBS}$, and stained with $5 \mu \mathrm{g} / \mathrm{ml}$ of the Hoechst 33342 fluorescent dye (Sigma-Aldrich) at $37^{\circ} \mathrm{C}$ for 90 minutes. Verapamil was added prior to Hoechst 33342 staining, to reliably ensure the identity and purity of the SP cells. Hoechst 33342-low cells were then analyzed and sorted using an ultraviolet laser cytometer (Beckman Coulter, Brea,
CA, USA). The data analysis was performed using FlowJo software (www.flowjo.com).

Polymerase chain reaction $(P C R)$. Total RNA was extracted from the EOC cells using TRIzol ${ }^{\circledR}$ reagent (Invitrogen Life Technologies, Carlsbad, CA, USA). A total of $1 \mu \mathrm{g}$ of RNA was used for each reverse transcription (RT) reaction. RT and PCR reactions were performed using the PrimeScript RT-PCR kit (Takara Bio, Inc., Otsu, Japan). Quantitative PCR (qPCR) analysis was performed using the ABI StepOne Plus Real-time PCR system (Applied Biosystems Life Technologies, Foster City, CA, USA) and SYBR Green Real-time PCR Master Mix (Takara Bio Inc.). The oligonucleotide primers used are listed in Tables I and II.

Western blot analysis. The cells were lysed using radioimmunoprecipitation assay buffer. The proteins were separated by denaturing electrophoresis using a $10 \%$ SDS-containing polyacrylamide gel, and then transferred onto polyvinyldene fluoride membranes (Invitrogen Life Technologies). The following rabbit anti-human antibodies were used: anti-Smad7 (1:700 dilution; Santa Cruz Biotechnology Inc., Dallas, TX, USA), anti-E-cadherin, anti-N-cadherin (1:1,000 dilutions; Cell Signaling Technology Inc., Danvers, MA, USA) and anti-GAPDH (Cell Signaling Technology Inc.). The membranes were incubated with the primary antibodies in Tris-buffered saline Tween ${ }^{\circledR} 20$ (TBST), containing $5 \%$ non-fat dry milk overnight at $4^{\circ} \mathrm{C}$, followed by an incubation with goat anti-rabbit immunoglobulin $\mathrm{G}$ (1;1,000; GE Healthcare Life Sciences, Chalfont, UK) in TBST, containing $2 \%$ nonfat dry milk at room temperature for $1 \mathrm{~h}$. ImageQuant LAS 4000 and Analysis Software (GE Healthcare Bio-Sciences, Pittsburgh, PA, USA) were used to quantify the intensity of the bands.

Transwell invasion assay. A transwell membrane $(8 \mu \mathrm{m}$ pore size, $6.5 \mathrm{~mm}$ diameter; Corning Costar, Corning, New York, NY, USA) was used. Matrigel ${ }^{\mathrm{TM}}$ (BD Biosciences, Franklin Lakes, NJ, USA) was melted on ice and diluted with cold RPMI-1640. The Matrigel ${ }^{\mathrm{TM}}(60 \mu \mathrm{l})$ was added into the top chamber and was gelated, following an incubation at $37^{\circ} \mathrm{C}$ for 5 hours. The cells were resuspended in RPMI-1640 at a concentration of 500,000 cells $/ \mathrm{ml}$. A $100 \mu \mathrm{l}$ cell suspension was plated into the Matrige ${ }^{\mathrm{TM}}$-coated top chambers and a total of $600 \mu 1$ RPMI-1640, containing 30\% FBS, was added to the bottom well. Following an incubation at $37^{\circ} \mathrm{C}$ for 48 hours, the membrane was stained with $0.1 \%$ crystal violet and observed under a Nikon upright microscope (Nikon Corporation, Tokyo, Japan). The experiments were repeated in triplicate, and the data are expressed as the means \pm standard error of the mean.

Wound healing assay. SK-OV-3 cells were seeded in 6-well plates at $1 \times 10^{5}$ per well. Following an overnight starvation with serum-free RPMI-1640, a single scratch wound was made on the cellular confluent monolayers, using a micropipette tip. Following incubation for 6, 12 and $24 \mathrm{~h}$, the migratory status of the cells was imaged using a Nikon microscope and analyzed with Image $\mathbf{J}$ software (National Institutes of Health, Bethesda, MA, USA). The migratory potential was 
Table I. Oligonucleotide primers used for reverse transcription-polymerase chain reaction analysis.

\begin{tabular}{|c|c|c|c|c|}
\hline Gene names & Accession & Size (bp) & Sequence & $\operatorname{Tm}\left({ }^{\circ} \mathrm{C}\right)$ \\
\hline hE-cadherin $(\mathrm{F})$ & NM_004360.3 & 425 & TCCСTTCCСТTGAGATGA & 46 \\
\hline hE-cadherin $(\mathrm{R})$ & & & GCCGATAGAATGAGACCCT & \\
\hline hN-cadherin $(\mathrm{F})$ & NM_001792.3 & 539 & TCGGGTAATCCTCCCAAAT & 52 \\
\hline hN-cadherin $(\mathrm{R})$ & & & CCACTGCCTTCATAGTCAAA & \\
\hline hVimentin $(F)$ & NM_003380.3 & 382 & GCCAGGCAAAGCAGGAGTC & 44 \\
\hline hVimentin (R) & & & TGGGTATCAACCAGAGGGAG & \\
\hline hSmad7 (F) & NM_005904.3 & 438 & ACAACCGCAGCAGTTACCC & 44 \\
\hline hSmad7 (R) & & & AAACGAGGACGAGAAGAAGAA & \\
\hline hSmad7 (F) & NM_005904.3 & 302 & СССТССТТАСТССАGATAСCC & 46 \\
\hline hSmad7 (R) & & & GCTGACTCTTGTTGTCCGAAT & \\
\hline hSmad3 (F) & NM_005902.3 & 106 & GCACCATCCGCATGAGCTTT & 44 \\
\hline hSmad3 (R) & & & TGCAAAGGCCCATTCAGGT & \\
\hline hSmad4 (F) & NM_005359.5 & 692 & AGCCATCGTTGTCCACTG & 48 \\
\hline hSmad4 (R) & & & GACCCAAACATCACCTTCAC & \\
\hline $\mathrm{h} \beta$-actin $(\mathrm{F})$ & NM_001614.3 & 159 & GCCCTGAGGCACTCTTCCA & 52 \\
\hline $\mathrm{h} \beta$-actin $(\mathrm{R})$ & & & TTGCGGATGTCCACGTCA & \\
\hline
\end{tabular}

F, forward; R, reverse; bp, base pairs; Tm, melting temperature; h, human.

Table II. Oligonucleotide primers used for real-time quantitative polymerase chain reaction analysis.

\begin{tabular}{|c|c|c|c|}
\hline Gene names & Accession & Size (bp) & Sequence \\
\hline hE-cadherin $(\mathrm{F})$ & NM_004360.3 & 114 & TTCCCTCGACACCCGATTC \\
\hline hE-cadherin $(\mathrm{R})$ & & & TAGGTGGAGTCCCAGGCGTA \\
\hline hN-cadherin $(\mathrm{F})$ & NM_001792.3 & 201 & ACAGTGGCCACCTACAAAGG \\
\hline hN-cadherin $(\mathrm{R})$ & & & CCGAGATGGGGTTGATAATG \\
\hline hVimentin $(F)$ & NM_003380.3 & 91 & CGAGGAGAGCAGGATTTCTC \\
\hline hVimentin (R) & & & GGTATCAACCAGAGGGAGTGA \\
\hline hSmad7 (F) & NM_005904.3 & 150 & CСССАTCACCTTAGCCGACTCTGC \\
\hline hSmad7 (R) & & & CCCAGGGGCCAGATAATT \\
\hline hSmad3 (F) & NM_005902.3 & 106 & GCACCATCCGCATGAGCTTT \\
\hline hSmad3 (R) & & & TGCAAAGGCCCATTCAGGT \\
\hline hSmad4 (F) & NM_005359.5 & 221 & CCATTTCCAATCATCCTGCT \\
\hline hSmad4 (R) & & & ACCTTTGCCTATGTGCAACC \\
\hline $\mathrm{h} \beta$-actin $(\mathrm{F})$ & NM_001614.3 & 159 & GCCCTGAGGCACTCTTCCA \\
\hline $\mathrm{h} \beta$-actin $(\mathrm{R})$ & & & TTGCGGATGTCCACGTCA \\
\hline
\end{tabular}

F, forward; R, reverse; bp, base pairs; h, human.

determined by measuring the distance between the wound edges. The results represent the percentage of the distance, following incubation for the specified times, relative to the original distance between the wound edges. Each experiment was repeated in triplicate and the data are expressed as the means \pm standard error of the mean.

Lentiviral transduction and Smad7 silence. A lentivirus driving the expression of green fluorescent protein (GFP) was transduced into SK-OV-3 cells to generate fluorescence-expressing cells. A Smad7-specific small hairpin RNA (shRNA) duplex (5'-ATTCGGACAACAAGAGTCA-3') and a vector control was cloned into U6-vshRNA-CMV-GFP lentiviral-based vectors (Shanghai JiKai Company, Shanghai, China). GFP-positive cells were detected using fluorescence-activated cell sorting (Beckman Coulter). Smad7 expression was detected by qPCR and western blot analysis.

Statistical analyses. The data are expressed as the means \pm standard error of the mean of $\geq 3$ independent experiments. The 
A

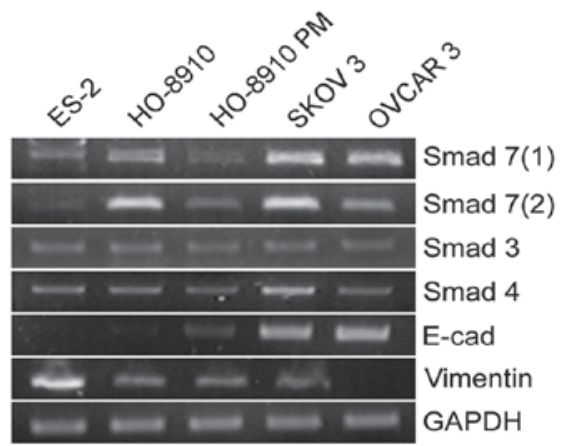

B

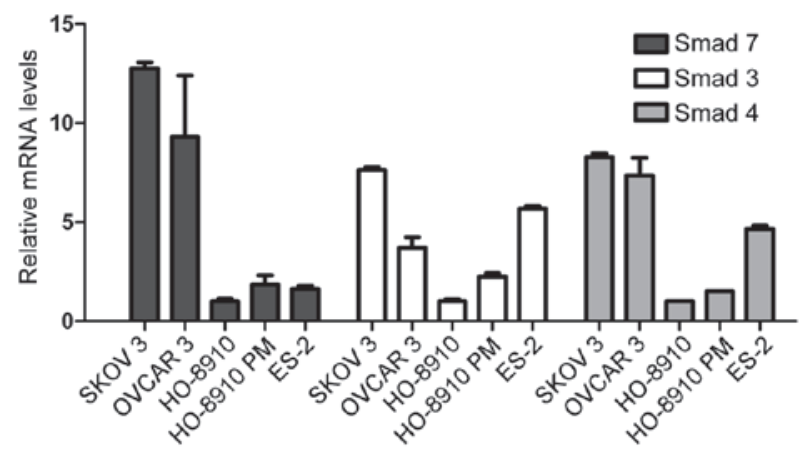

C

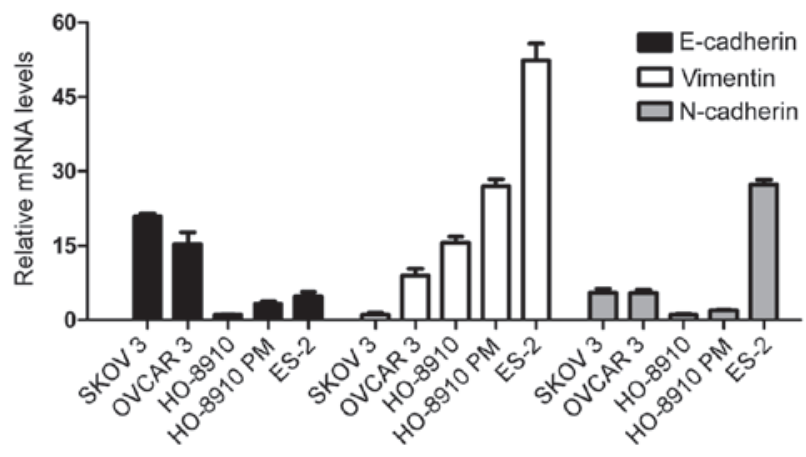

Figure 1. Higher Smad7 mRNA expression was detected in ovarian cancer cells with epithelial phenotypes. The relative mRNA expression levels of Smad3, Smad4, Smad7, E-cadherin, N-cadherin and Vimentin in RNA samples from ES-2, HO-8910, HO-8910PM, SK-O-V3 and OVCAR-3 epithelial ovarian cancer cell lines were analyzed by (A) reverse transcription-polymerase chain reaction and (B and $\mathrm{C})$ quantitative PCR.

significance of the differences between the mean values was determined using a two-tailed Student's t-test. A P $<0.05$ was considered to indicate a statistically significant difference.

\section{Results}

Smad7 is expressed at a higher level in the epithelial growth-patterned SK-OV-3 cell line. To observe the functions of the TGF- $\beta / S$ mad signaling pathway in EOC, the relative mRNA expression levels of Smad3, Smad4 and Smad7 were determined in SK-OV-3, ES-2, OVCAR-3, HO-8910 and HO-8910PM cells. As shown in Fig. 1A and B, Smad3, Smad4 and Smad7 were more highly expressed in the SK-OV-3 cells, as compared with the other ovarian cancer cell lines. Among the Smads, Smad7 was the most elevated. These results indicate that $\mathrm{Smad} 7$ may have a unique func- tion in the TGF- $\beta /$ Smad signaling pathway and may exert an effect on the regulation of EMT in EOC cells.

To further distinguish whether the expression of the Smads was associated with an epithelial/mesenchymal phenotype, an EMT-related gene expression profile, including E-cadherin, $\mathrm{N}$-cadherin and Vimentin, was compared between the five cell lines (Fig. 1A and C). The epithelial marker E-cadherin, was more highly expressed in the SK-OV-3 cells, as compared with the other cell lines. The mesenchymal marker $\mathrm{N}$-cadherin, was more highly expressed in the ES-2 cells. In addition, SK-OV-3 and OVCAR-3 cells exhibited a more distinct epithelial round-clonal growth pattern and exhibited abundant intercellular junctions, whereas ES-2 cells exhibited a sparse fibroblast growth pattern and a branched cytoplasm.

These results indicate that, although the five cell lines were all generated from the tissue samples of patients with EOC, the SK-OV-3 cells had a more epithelial behavior and the ES-2 cells more mesenchymal. Furthermore, the relative expression level of Smad7 was highest in the SK-OV-3 cells.

Smad7 is overexpressed in ovarian cancer stem-like $S P$ cells. Ovarian cancer tumors are heterogeneous and contain CSCs that are able to self-renew and are known to be essential to tumorigenesis and progression (22). EOC stem-like SP cells were shown to exhibit an epithelial phenotype and a reduced expression of cell adhesion molecules. Non-SP cells forfeited the CSC properties and exhibited a more mesenchymal phenotype (10). To explore the underlying association between Smad7 and ovarian CSCs, a Hoechst 33342-effluxing assay was used to detect and enrich SP cells from EOC HO-8910PM cells (Fig. 2A). The SP cells were shown to exhibit much higher relative mRNA expression levels of Smad7, as compared with the non-SP cells (Fig. 2B). Because of the epithelial origin of ovarian cancer SP cells, Smad7 exhibited an increased expression in the cells with an epithelial phenotype, either in ovarian CSCs or in the EOC cell line. These results indicate that Smad7 may have an important role in the maintenance of the epithelial phenotype.

TGF- $\beta 1$ upregulates the expression of Smad7 in EOC cells. TGF- $\beta 1$ is a fundamental determinant of cell migration in wound healing, inflammation and tumorigenesis (22). It has also been identified as the main factor in the progression of an invasive phenotype in epithelial tumors (23). To determine the association between TGF- $\beta 1$ stimulation and Smad7 expression in EOC, SK-OV-3 cells were cultured in the presence of TGF- $\beta 1$ for two days. Following TGF- $\beta 1$ stimulation, SK-OV-3 cells exhibited a reduced relative expression of E-cadherin and an increased relative expression of $\mathrm{N}$-cadherin, at both the mRNA and protein level (Fig. 3A-C). These results imply an E-cadherin to N-cadherin switch (EN switch). Furthermore, Smad7 was upregulated during TGF- $\beta 1$ stimulation, in accordance with the increase of the meshenchymal marker N-cadherin (Fig. 3D). As a downstream inhibitor of TGF- $\beta 1$-induced EMT, the increased expression of Smad7 during TGF- $\beta 1$ stimulation may be a result of a feedback mechanism in EOC, reversing the EN switch to MET. 
A

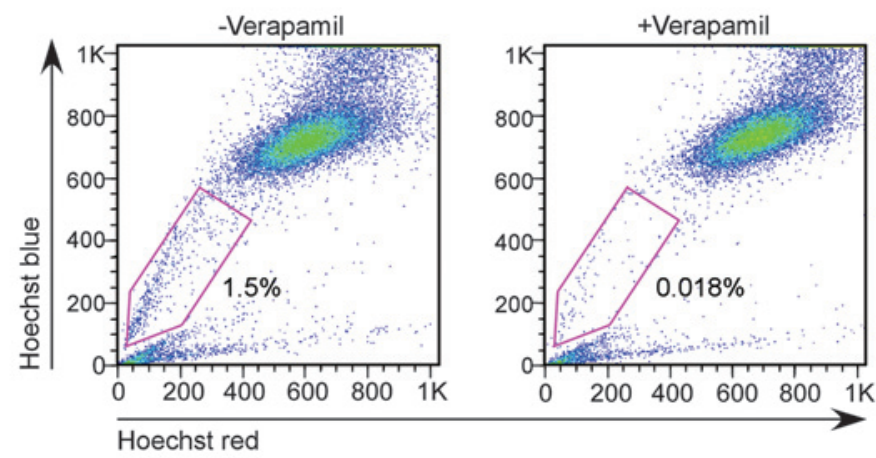

B

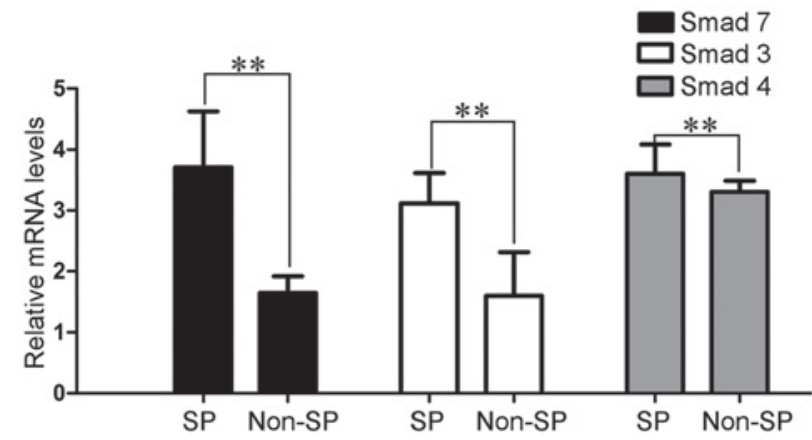

Figure 2. High Smad7 expression was observed in ovarian cancer stem-like side population (SP) cells. (A) The percentage of the subset of SP in the total HO-8910PM ovarian cancer cell population was determined by flow cytometry following Hoechst 33342 staining (left panel). The Hoechst 33342-low cell population was shown to decrease in the presence of Verapamil (right panel). (B) The relative expression levels of Smad3, Smad4 and Smad7, in SP cells, were decreased in non-SP cells as determined by quantitative polymerase chain reaction. The data represent the means \pm standard error of the means $\mathrm{P}<0.05$, ${ }^{* *} \mathrm{P}<0.01$, as compared with SP cells.

A

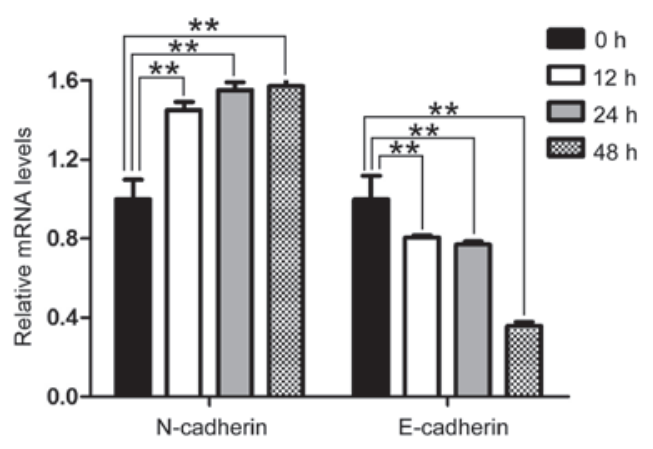

B

C

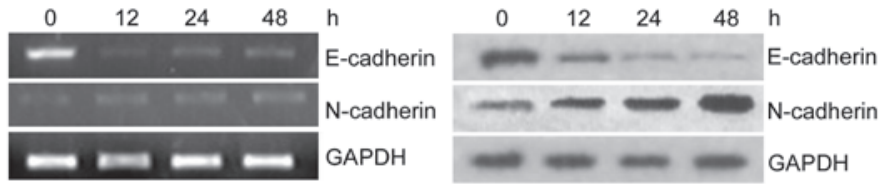

D

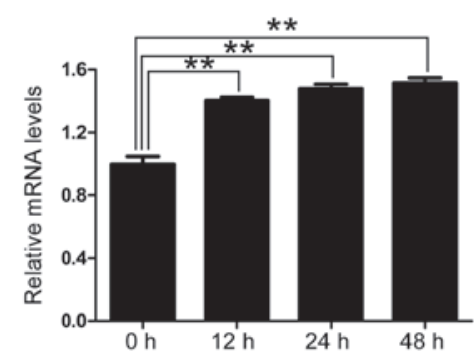

Figure 3. Transforming growth factor $\beta 1$ (TGF- $\beta 1$ ) induced epithelial-mesenchymal transition and increased Smad7 expression in ovarian cancer cells. The expression profiles of E-cadherin and N-cadherin, in SK-OV-3 cells during TGF- $\beta 1$ stimulation $(12,24,48 \mathrm{~h})$ relative to the unstimulated level $(0 \mathrm{~h})$ were determined by (A) quantitative polymerase chain reaction (qPCR); (B) reverse transcription-PCR; and (C) western blotting. (D) The relative mRNA expression levels of Smad7, during TGF- $\beta 1$ stimulation $(12,24,48 \mathrm{~h})$, relative to the unstimulated level $(0 \mathrm{~h})$ were determined by qPCR. The data represent the means \pm standard error of the mean; ${ }^{* *} \mathrm{P}<0.01$, as compared with $0 \mathrm{~h}$. 
A

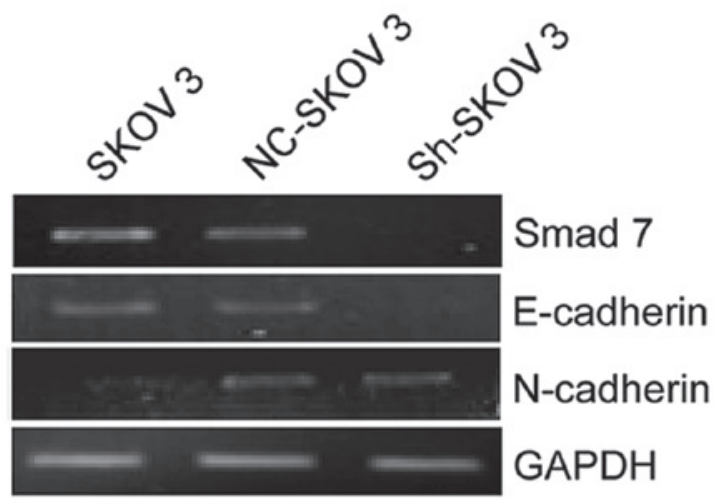

B

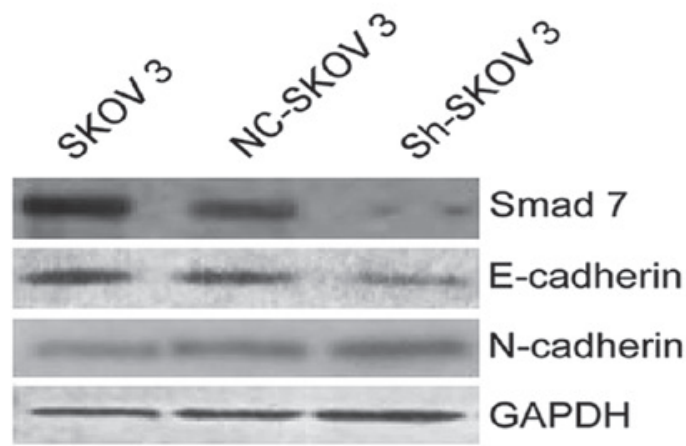

D

C
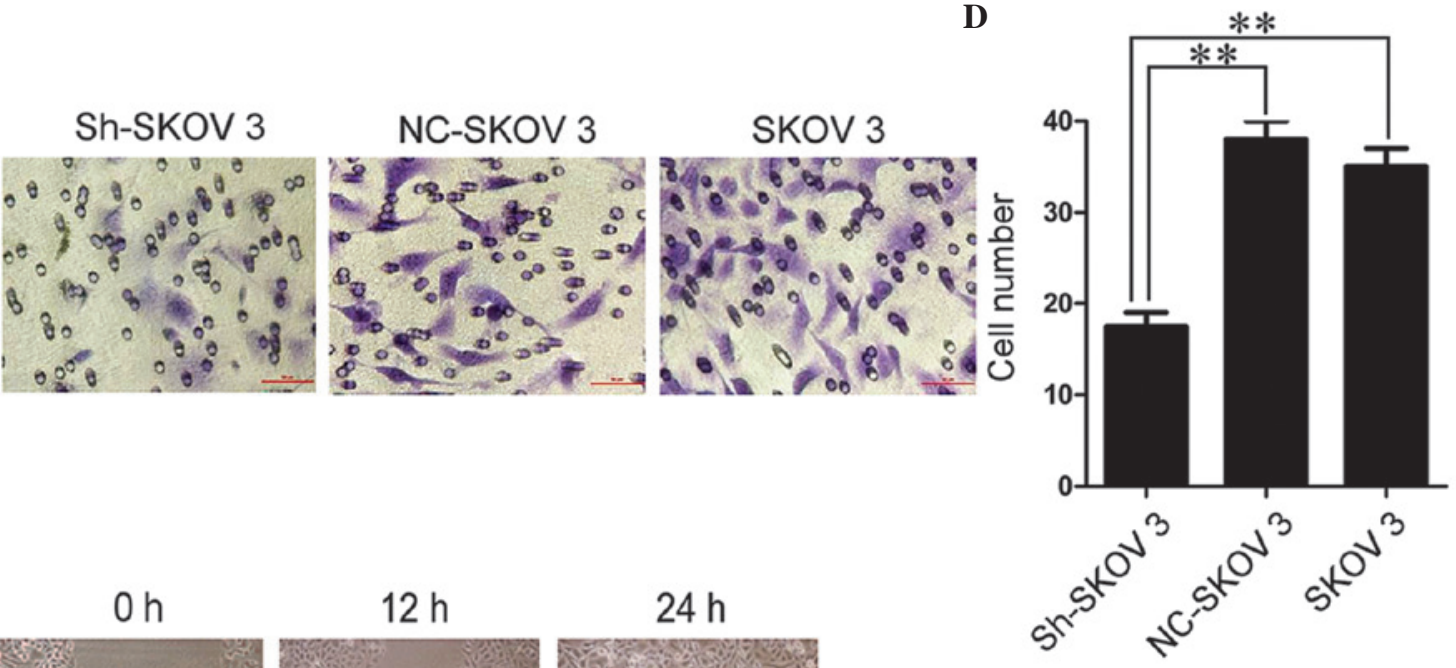

$\mathbf{E}$

$\mathrm{Oh}$
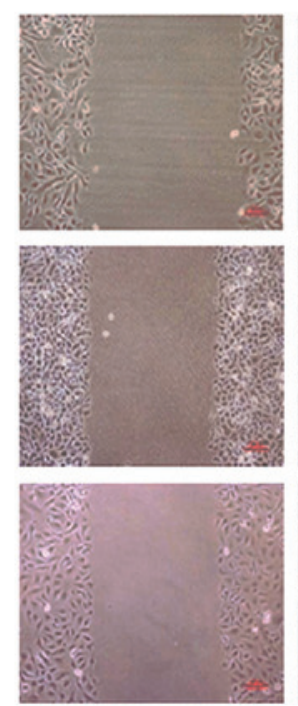

$12 \mathrm{~h}$
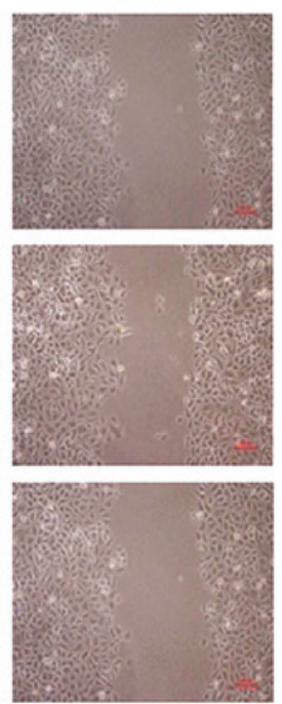

$24 \mathrm{~h}$

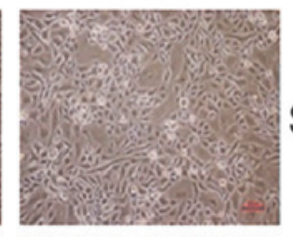

Sh-SKOV 3 F

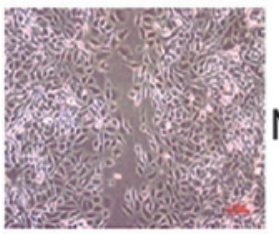

NC-SKOV 3

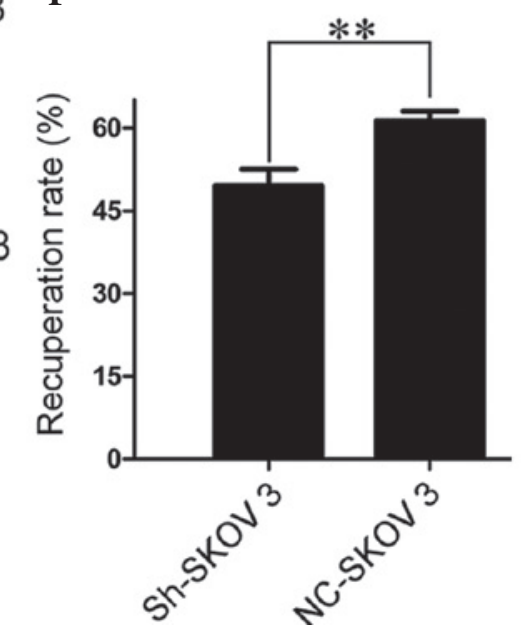

Figure 4. Downregulation of Smad7 enhanced the ovarian cancer cell transformation into the mesenchymal phenotype, but inhibited their invasive and migratory capacity. Untransduced SK-OV-3 cells, and cells transduced with control vector or Smad7 small hairpin (shRNA (shSmad7) were analyzed by (A) quantitative polymerase chain reaction, and (B) western blotting to determine the mRNA and protein expression levels of E-cadherin, N-cadherin and Smad7. (C) Untransduced SK-OV-3 cells, and SK-OV-3 cells transduced with control vector and shSmad7 were suspended in serum-free media and plated onto the top chamber of a Matrigel-covered microspore transwell. The cells that migrated into the membrane were stained with crystal violet and counted under a upright microscope (magnification, $x 10$ ). (D) Results of the trasnwell invasion assay are graphically expressed as the mean number of invasive cells \pm standard error of the mean; ${ }^{* *} \mathrm{P}<0.01$. (E) Confluent monolayers of untransduced SK-OV-3 cells and SK-OV-3 cells transduced with control vector and shSmad7 were wounded. The migratory distance of the wounded cells was imaged under a microscope after 12 and $24 \mathrm{~h}$ (magnification, $\mathrm{x} 10$ ). (F) The distance between the wound edges were measured after 12 hours and was compared with the original. The results are graphically expressed as the mean percentage of migratory distance \pm standard error of the mean; ${ }^{* *} \mathrm{P}<0.01$, as compared with sh-SKOV3.

Silencing of Smad7 promotes EMT but reduces the invasive and migratory capabilities of EOC cells. Both the process of EMT and the gene expression of Smad7 were stimulated by
TGF- $\beta 1$, therefore the present study further aimed to determine the specific operative function of Smad7 on EMT. A shRNA lentiviral construct specifically targeting the human Smad7 
gene was generated and transduced into SK-OV-3 cells. The relative expression of $\mathrm{Smad} 7$ was efficiently inhibited at both the mRNA and protein level (Fig. 4A and B). As predicted, the process of EMT was accelerated by the inhibition of Smad7. The mRNA encoding epithelial marker, E-cadherin, was downregulated in Smad7 shRNA (shSmad7)-transduced SKOV3 cells, whereas the mRNA encoding mesenchymal marker, N-cadherin, was upregulated (Fig. 4A and B). These results confirm that Smad7 inhibited EMT, and repression of Smad7 may induce cellular mesenchymal transformation in tumors.

To evaluate the variation of tumor biological characteristics in shSmad7-SKOV3 cells, transwell invasion and wound healing assays were performed to estimate the cellular invasive and migratory capabilities, respectively. As compared with the vector control, ectopic deletion of Smad7 led to a markedly reduced number of infiltrating and migrating cells in shSmad7-SKOV3 cells (Fig. 4C-F). These results indicate that Smad7 exerts its function on cellular motility and metastatic capacity in EOC development. This is in contrast with the previous findings that shSmad7-SKOV3 cells sustained a mesenchymal phenotype (Fig. 4A and B). The process of EMT is complex and is associated with numerous signaling pathways (24), therefore other EMT molecular mechanisms may be compensating for the lack of Smad7 expression, in order to maintain the invasiveness of the EOC cells.

\section{Discussion}

EMT has been indicated as a dynamic process required for cellular remodeling during embryogenesis, wound healing and the acquisition of malignant traits (25). It also leads to the invasion and metastasis of tumor cells, as the result of alternation of cell-cell adhesion, cell polarity and cell-extracellular matrix interaction (5). Its reverse process, MET, may accelerate neoplasm development and tumor colonization once metastatic tumor cells have grown at the distant sites (12-14). Numerous metastatic initiating elements are required to induce tumor cell migration, invasion, intravasation and activation of angiogenesis $(4,6)$, among which Smad7 has contradictory effects on cancer progression and metastasis as previously described (17-19). In the intracellular TGF- $\beta$ signaling pathway, the polypeptide Smad7 serves to accommodate negative feedback $(15,23)$. As previously reported, Smad7 is the predominant inhibitor in TGF- $\beta$-induced EMT, through the binding of the TGF- $\beta 1$ receptor and obstructing the phosphorylation of Smad2 and Smad3. A pre-existing nuclear pool of Smad7 can also be mobilized by TGF- $\beta 1$. Hence it constitutes a delicate negative feedback loop, which is associated with sustaining the balance of TGF- $\beta /$ Smad-regulated EMT $(15,26)$.

Tumor cell migration involves complex pathways which are spatially and temporally associated with the transformation of the cytoskeleton and morphology of cells (27). It has been previously suggested that $\mathrm{Smad} 7$ is associated with the TGF- $\beta$-triggered cytoskeletal responses of migrating cells, through local regulation of polarity complexes (28). As a feedback regulator in TGF- $\beta /$ Smad-modulated EMT, the aberrant expression of Smad7 can induce malignant cellular progression and is considered to be oncogenic $(22,29)$. However, it has been observed, in numerous cases, that $\mathrm{Smad} 7$ may have an opposite effect, according to the cancer type. Overexpression of Smad7 has previously been shown to hinder the advancement of brain (30), breast (31), colorectal (32), nasopharyngeal (17), cervical (18), bone and lung $(30,31)$ cancers, both in vitro and in vivo. Conversely, Smad7 induced malignant processes of squamous cell (33) and colon carcinoma (34), especially tumorigenicity and metastasis recurrence. Despite the identified status of Smad7 in numerous cancers, the functions of Smad7 in EOC pathogenesis remains undefined.

In the present study, the transcription of Smad3, Smad4 and Smad7 were assessed in five EOC cell lines. The relative expression of Smad7 was shown to be highest in the SK-OV-3 cells, which presented a more epithelial and less mesenchymal phenotype. EOC tumors are heterogeneous and whether ovarian cancer cells are of a epithelial or mesenchymal origin remains unknown. A previous study confirmed that ovarian cancer stem-like SP cells were of epithelial origin (10). Furthermore, Smad7 was identified as being more highly expressed in the epithelial phenotypic SP cells, as compared with the mesenchymal phenotypic non-SP cells. These data are consistent with the hypothesis that $\mathrm{Smad} 7$ is associated with the epithelial ovarian tumor initiator CSCs. Downregulation of Smad7 by a shSmad7 lentiviral vector, resulted in the decreased expression of E-cadherin and increased expression of $\mathrm{N}$-cadherin. These results indicate that as an inhibitor in the process of EMT, Smad7 has an important role in the maintenance of the epithelial phenotype in ovarian cancer cells. The particular mechanism by which Smad7 affects the endogenous phenotypic transformation requires further investigation.

To induce EMT, SK-OV-3 cells were stimulated with TGF- $\beta 1$. The cells gradually converted from a round epithelial pattern to a long fusiform mesenchymal shape; reducing epithelial marker expression levels and fortifying the mesenchymal phenotype. Meanwhile, the expression of Smad7 was also upregulated. It may be hypothesized that during TGF- $\beta$-stimulated EMT, the increased expression of Smad7 increasingly expressed at a notable level, may be the result of a negative feedback mechanism (35).

In addition, the shSmad7-SKOV3 cells developed a mesenchymal profile of cell surface makers. They exhibited a more mesenchymal circular shape, with simultaneous $\mathrm{N}$-cadherin upregulation and E-cadherin downregulation. However, the invasive and metastatic capacity of the shSmad7-SKOV3 cells was markedly reduced. These results indicate that the Smad-associated pathway is not the sole molecular mechanism participating in the regulation of EMT. Numerous other signaling pathways, including $\mathrm{NF}-\kappa \mathrm{B}$, Wnt and Notch (24), may compensate for the suppression of Smad7, in order to maintain the invasive behavior of the cancer cells. By restoring the epithelial phenotype and invasive behavior of ovarian cancer cells, Smad7 may contribute to the MET process in EOC, which facilitates the growth of epithelial tumor cells at metastatic sites.

In conclusion, the findings of the present study indicate a negative regulatory mechanism between Smad7 and EMT in EOC. Smad7 has an important role in retaining the epithelial phenotype of ovarian cancer cells, and may induce tumor cell invasion and migration. This bilateral behavior may exert its function when ovarian cancer cells reverse EMT to MET, 
which is necessary for the colonization of metastatic sites. The underlying mechanisms that regulate TGF- $\beta /$ Smad signaling in EOCs require further elucidation.

\section{Acknowledgements}

This study was supported by the National Natural Science Foundation of China (no. 31371452; Hua Jiang) and the Foundations from Science and Technology Commission of Shanghai Municipality (nos. 11JC1401501, 12410710100; Hua Jiang).

\section{References}

1. Lengyel E: Ovarian cancer development and metastasis. Am J Pathol 177: 1053-1064, 2010.

2. Peinado H, Olmeda D and Cano A: Snail, Zeb and bHLH factors in tumour progression: an alliance against the epithelial phenotype? Nat Rev Cancer 7: 415-428, 2007.

3. Iwatsuki M,Mimori K, Yokobori T, et al: Epithelial-mesenchymal transition in cancer development and its clinical significance. Cancer Sci 101: 293-299, 2010.

4. Condeelis J and Segall JE: Intravital imaging of cell movement in tumours. Nat Rev Cancer 3: 921-930, 2003.

5. Nisticò P, Bissell MJ and Radisky DC: Epithelial-mesenchymal transition: general principles and pathological relevance with special emphasis on the role of matrix metalloproteinases. Cold Spring Harb Perspect Biol 4, 2012.

6. Birchmeier W and Behrens J: Cadherin expression in carcinomas: role in the formation of cell junctions and the prevention of invasiveness. Biochim Biophys Acta 1198: 11-26, 1994.

7. Elloul S, Elstrand MB, Nesland JM, et al: Snail, Slug, and Smad-interacting protein 1 as novel parameters of disease aggressiveness in metastatic ovarian and breast carcinoma. Cancer 103: 1631-1643, 2005.

8. Jin H, Yu Y, Zhang T, et al: Snail is critical for tumor growth and metastasis of ovarian carcinoma. Int J Cancer 126: 2102-2111, 2010

9. AhmedN,ThompsonEW andQuinnMA:Epithelial-mesenchymal interconversions in normal ovarian surface epithelium and ovarian carcinomas: an exception to the norm. J Cell Physiol 213: 581-588, 2007.

10. Jiang H, Lin X, Liu Y, et al: Transformation of epithelial ovarian cancer stemlike cells into mesenchymal lineage via EMT results in cellular heterogeneity and supports tumor engraftment. Mol Med 18: 1197-1208, 2012.

11. Thiery JP, Acloque H, Huang RY and Nieto MA: Epithelial-mesenchymal transitions in development and disease. Cell 139: 871-890, 2009.

12. Elloul S, Vaksman O, Stavnes HT, Trope CG, Davidson B and Reich R: Mesenchymal-to-epithelial transition determinants as characteristics of ovarian carcinoma effusions. Clin Exp Metastasis 27: 161-172, 2010

13. Polyak K and Weinberg RA: Transitions between epithelial and mesenchymal states: acquisition of malignant and stem cell traits. Nat Rev Cancer 9: 265-273, 2009.

14. Wells A, Yates C and Shepard CR: E-cadherin as an indicator of mesenchymal to epithelial reverting transitions during the metastatic seeding of disseminated carcinomas. Clin Exp Metastasis 25: 621-628, 2008.

15. Valcourt U, Kowanetz M, Niimi H, Heldin CH and Moustakas A: TGF-beta and the Smad signaling pathway support transcriptomic reprogramming during epithelial-mesenchymal cell transition. Mol Biol Cell 16: 1987-2002, 2005.
16. Ikushima $\mathrm{H}$ and Miyazono K: Biology of transforming growth factor- $\beta$ signaling. Curr Pharm Biotechnol 12: 2099-2107, 2011.

17. Xiao J, Xiang Q, Xiao YC, et al: The effect of transforming growth factor-beta1 on nasopharyngeal carcinoma cells: insensitive to cell growth but functional to TGF-beta/Smad pathway. J Exp Clin Cancer Res 29: 35, 2010.

18. Hariharan R, Babu JM, P R and Pillai MR: Mutational analysis of Smad7 in human cervical cancer. Oncol Rep 21: 1001-1004, 2009.

19. Luo X, Xu J and Chegini N: The expression of Smads in human endometrium and regulation and induction in endometrial epithelial and stromal cells by transforming growth factor-beta. J Clin Endocrinol Metab 88: 4967-4976, 2003.

20. Shenhua X, Lijuan Q, Hanzhou N, et al: Establishment of a highly metastatic human ovarian cancer cell line (HO-8910PM) and its characterization. J Exp Clin Cancer Res 18: 233-239, 1999.

21. Goodell MA, Brose K, Paradis G, Conner AS and Mulligan RC: Isolation and functional properties of murine hematopoietic stem cells that are replicating in vivo. J Exp Med 183: 1797-1806, 1996.

22. Drabsch Y and ten Dijke P: TGF- $\beta$ signalling and its role in cancer progression and metastasis. Cancer Metastasis Rev 31: 553-568, 2012.

23. Horiguchi K, Shirakihara T, Nakano A, Imamura T, Miyazono K and Saitoh M: Role of Ras signaling in the induction of snail by transforming growth factor-beta. J Biol Chem 284: 245-253, 2009.

24. Moustakas A and Heldin $\mathrm{CH}$ : Signaling networks guiding epithelial-mesenchymal transitions during embryogenesis and cancer progression. Cancer Sci 98: 1512-1520, 2007.

25. Singh A and Settleman J: EMT, cancer stem cells and drug resistance: an emerging axis of evil in the war on cancer. Oncogene 29: 4741-4751, 2010.

26. Itóh S, Landström M, Hermansson A, et al: Transforming growth factor betal induces nuclear export of inhibitory Smad7. J Biol Chem 273: 29195-29201, 1998.

27. Ridley AJ, Schwartz MA, Burridge K, et al: Cell migration: integrating signals from front to back. Science 302: 1704-1709, 2003.

28. Ekman M, Mu Y, Lee SY, et al: APC and Smad7 link TGF $\beta$ type I receptors to the microtubule system to promote cell migration. Mol Biol Cell 23: 2109-2121, 2012.

29. Pittman AM, Naranjo S, Webb E, et al: The colorectal cancer risk at $18 \mathrm{q} 21$ is caused by a novel variant altering SMAD7 expression. Genome Res 19: 987-993, 2009.

30. Javelaud D, Mohammad KS, McKenna CR, et al: Stable overexpression of Smad7 in human melanoma cells impairs bone metastasis. Cancer Res 67: 2317-2324, 2007.

31. Azuma H, Ehata S, Miyazaki H, et al: Effect of Smad7 expression on metastasis of mouse mammary carcinoma JygMC(A) cells. J Natl Cancer Inst 97: 1734-1746, 2005.

32. Rizzo A, Waldner MJ, Stolfi C, et al: Smad7 expression in T cells prevents colitis-associated cancer. Cancer Res 71: 7423-7432, 2011.

33. Liu X, Lee J, Cooley M, Bhogte E, Hartley S and Glick A: Smad7 but not Smad6 cooperates with oncogenic ras to cause malignant conversion in a mouse model for squamous cell carcinoma. Cancer Res 63: 7760-7768, 2003.

34. Halder SK, Beauchamp RD and Datta PK: Smad7 induces tumorigenicity by blocking TGF-beta-induced growth inhibition and apoptosis. Exp Cell Res 307: 231-246, 2005.

35. Gratchev A, Kzhyshkowska J, Kannookadan S, et al: Activation of a TGF-beta-specific multistep gene expression program in mature macrophages requires glucocorticoid-mediated surface expression of TGF-beta receptor II. J Immunol 180: 6553-6565, 2008. 